\title{
CORRIGENDUM
}

\section{Metabolic and physiologic improvements from consuming a paleolithic, hunter-gatherer type diet}

LA Frassetto, M Schloetter, M Mietus-Synder, RC Morris and A Sebastian

European Journal of Clinical Nutrition (2015) 69, 1376; doi:10.1038/ejcn.2015.193

Correction to: European Journal of Clinical Nutrition (2009) 63, 947-955; doi:10.1038/ejcn.2009.4

Since the publication of the above article the authors have identified errors in the values listed in Table 3 for 'Fasting glucose, $\mathrm{mmol} / \mathrm{l}$. Errors were introduced when transferring data from original to SI units.
The corrected table is shown here. The authors have confirmed that the changes have no impact on the statistics or discussion presented.

The authors apologise for any inconvenience caused.

Table 3. Effect of the paleolithic diet on metabolic variables

\begin{tabular}{|c|c|c|c|c|c|}
\hline Category & Variable & Usual diet & Paleolithic diet & $\%$ change & $P$ value \\
\hline \multirow[t]{5}{*}{ Lipids } & Total cholesterol, $\mathrm{mmol} / \mathrm{l}$ & $4.7 \pm 0.9$ & $4.0 \pm 0.7$ & -16 & 0.007 \\
\hline & $\mathrm{HDL}, \mathrm{mmol} / \mathrm{I}$ & $1.3 \pm 0.2$ & $1.3 \pm 0.3$ & +4 & NS \\
\hline & $\mathrm{LDL}, \mathrm{mmol} / \mathrm{l}$ & $3.0 \pm 0.7$ & $2.3 \pm 0.6$ & -22 & 0.003 \\
\hline & $\mathrm{VLDL}, \mathrm{mmol} / \mathrm{l}$ & $0.4 \pm 0.2$ & $0.3 \pm 0.1$ & -35 & 0.01 \\
\hline & Triglycerides, mmol/l & $0.9 \pm 0.4$ & $0.6 \pm 0.1$ & -35 & 0.01 \\
\hline \multirow[t]{2}{*}{ Fasting insulin and glucose } & Fasting insulin, pmol/l & $69 \pm 63$ & $21 \pm 7$ & -68 & 0.07 \\
\hline & Fasting glucose, $\mathrm{mmol} / \mathrm{l}$ & $5.1 \pm 1.8$ & $4.8 \pm 0.4$ & -5 & NS \\
\hline \multirow[t]{2}{*}{ OGTT } & Insulin AUC, pmol h/l & $533 \pm 222$ & $361 \pm 194$ & -39 & 0.006 \\
\hline & $\mathrm{HOMA}^{\mathrm{a}}$ & $2.3 \pm 2.3$ & $0.7 \pm 0.3$ & -72 & 0.07 \\
\hline
\end{tabular}

${ }^{\mathrm{a}}$ Equation for $\mathrm{HOMA}=[$ fasting insulin $\times$ fasting glucose $] / 22.4$. 